Journal of the Mathematical Society of Japan Vol. 1, No. 2, July, 1949.

\title{
A boundary value problem of some special ordinary differential equations of the second order.
}

\author{
Shigeo SASAKI.
}

(Received Oct. 2, 1947)

\section{$\S 1$. Statement of the problem.}

1. Among various results concerning the behaviour of geodesics in the large, there are many theorems which can be stated without the concept of length. They seem to be only properties in the large of integral curves of a system of differential equations of the second order. Hence, some of them may be generalized to the geometry of paths in the large.

Now, the first-problem in the theory of geodesics in the large is that "Given any two points on a surface, can they always be bound by a geodesic?" From the point of view stated above, there then arises the following problem: Given any two points in a plane, can they always be bound by a path? A path is, by definition, an integral curve of a system of ordinary differential equations of the second order of the following type

$$
\begin{aligned}
& \ddot{x}=A_{1} \dot{x}^{2}+2 B_{1} \ddot{x} \dot{y}+C_{1} \dot{y}^{2}, \\
& : \ddot{y}=A_{2} \dot{x}^{2}+2 B_{2} \dot{x} \dot{y}+C_{2} \dot{y}^{2},
\end{aligned}
$$

where dots denote derivatives with respect to a parameter $t$, and $A_{1}, B_{1}, \ldots$ $\ldots, C_{2}$ denote continuous functions of $x$ and $y$. Putting $x=x^{1}, y=x^{2}$, the set of equations (1) are usually written as

$$
\ddot{x}^{i}+\Gamma_{j k}^{i} \dot{x}^{j} \dot{x}^{k}=0, \quad(i, j, k=1,2)
$$

$\Gamma_{j k}^{i}$ being called parameters of an affine connexion.

The answer of the problem is in general negative. But, it is desirable to know in what manner it becomes impossible, in other. words, the behaviour of integral curves.

In this paper we shall confine ourselves to the simplest case where $A_{1}$, $B_{1}, \ldots \ldots, C_{2}$ are all real constants. Our result may be stated as follows:

Theorem. Let there be given a system of differential equations of the form (1) with real constant corfficients $A_{1}, B_{1}, \ldots \ldots, C_{2}$. Then they. can be classified into two types. For one of them any two points in plane can be. 
bound always by a path, and for another any point in plane can be bound by a path with those and only those points which lie between certain parallel lines at equal distance from the first point.

2. If we eliminate the parameter $t$, the set of equations (1) reduces to a single equation

$$
y^{\prime \prime}+C_{1} y^{\prime 3}+\left(2 B_{1}-C_{2}\right) y^{\prime 2}+\left(A_{1}-2 B_{2}\right) y^{\prime}-A_{2}=0
$$

where dashes denote derivatives with respect to $x$. Hence our problem may. be regarded also as a boundary value problem of a differential equation of the second order of the type

$$
y^{\prime \prime}+P_{3}\left(y^{\prime}\right)=0
$$

where $P_{3}\left(y^{\prime}\right)$ denotes a polynomal of the third order of $y^{\prime}$ with constant coefficients.

The equation (3) will be also used to give a proof of our theorem.

\section{§ 2. Canonical forms of the differential equations.}

3. The equation (3) shows that system of paths depends only on the values $C_{1}, A_{2}$ and the differences $2 B_{1}-C_{2}, A_{1}-2 B_{2}$. Hence we can change the coefficients of $(\mathrm{T})$ without aitering paths. This fact corresponds to the projective change of affine connexion in the geometry of paths, which is analytically expressed by

$$
\bar{\Gamma}_{j k}^{\prime i}=I_{j k}^{i}+\delta_{j}^{i} \psi_{k}+\delta_{j}^{i} \phi_{j}^{\prime}, \quad \cdot
$$

where $\psi_{1}$ and $\phi_{2}$ are arbitrary functions of $x$ and $y$. Thus we can put any given set of equations (1) in the form

$$
\begin{aligned}
& \ddot{x}=A_{1} \dot{x}^{2}+C_{1} \dot{y}^{2}, \\
& \ddot{y}=A_{2} \dot{x}^{2}+C_{2} \dot{y}^{2},
\end{aligned}
$$

without altering the system of paths.

Now consider the transformation of coordinates :

$$
x^{*}=\lambda x, \quad y^{*}=\mu y,
$$

where $\lambda ; \mu$ denote non zero constants. Then the set of equations (5) reduces to . 


$$
\begin{aligned}
& \ddot{x^{*}}=\frac{A_{1}}{\lambda} \dot{x}^{* 2}+\frac{\lambda C_{1}}{\mu^{2}} \dot{y}^{* 2}, \\
& \ddot{y}^{*}=\frac{\mu A_{2}}{\lambda^{2}} \dot{x}^{* 2}+\frac{C_{2}}{\mu} \dot{y}^{* 2} .
\end{aligned}
$$

Taking $\lambda$ and $\mu$ suitably, we can reduce (5) to the following "canonical forms ".

Case I. $C_{1} \neq 0, A_{2} \neq 0$. If we put

$$
\lambda=\sqrt[3]{.{ }_{1} A_{2}^{2}}, \quad \mu=\sqrt[3]{C_{1}^{2} A_{2}},
$$

$C_{1}^{*}$ and $A_{2}{ }^{*}$ become both 1 . Hence (6) reduces to

$$
\begin{aligned}
& \ddot{x}=a \dot{x}^{2}+\dot{y}^{2}, \\
& \ddot{y}=\dot{x}^{2}+b \dot{y}^{2},
\end{aligned}
$$

where $a$ and $b$ may be zero.

Case II. $C_{1}=0, A_{2} \neq 0, C_{2} \neq 0$. Take the sign of $\mu$ so that $\mu A_{2}$ becomes positive, and define the values of $\lambda$ and $\mu$ by the relations

$$
\lambda=\sqrt{\mu A}, \quad\left|\frac{C_{2}}{\mu}\right|=1,
$$

then $(6)$ reduces to

$$
\begin{aligned}
& \ddot{x}=a \dot{x}^{2}, \\
& \ddot{y}=\dot{x} \pm \dot{y}^{2},
\end{aligned}
$$

where a may be 'zero.

Case III. $C_{1}=0, A_{2} \neq 0, C_{2}=0, A_{1} \neq 0$. It is clear that $(6)$ reduces in this case to

$$
\begin{aligned}
& \ddot{x}=\dot{x}^{2}, \\
& \ddot{y}=\dot{x}^{2} .
\end{aligned}
$$

Case IV. $C_{1}=0, A_{2} \neq 0, C_{2}=0, A_{1}=0$. (6) reduces in this case to

$$
\begin{aligned}
& \ddot{x}=0, \\
& \ddot{y}=\dot{x}^{2} .
\end{aligned}
$$


In the cases II, III, IV, we have treated the case where $C_{1}=0, A_{2} \neq 0$. There may happen also the case where $A_{2}=0, C_{1} \neq 0$. Howèver, the latter is equivalent to the former as is easily seen by interchanging $x$ and $y$. Hence we can omit it.

- Case V. $C_{1}=0, A_{2}=0, A_{1} \neq 0, C_{2} \neq 0$. In this case (6) reduces to

$$
\begin{aligned}
& \ddot{x}=\dot{x}^{2}, \\
& \ddot{y}=\dot{y}^{2} .
\end{aligned}
$$

Case VI. $C_{1}=0, A_{2}=0, A_{1}=0, C_{2} \neq 0$.

$$
\begin{aligned}
& \ddot{x}=0, \\
& \ddot{y}=\dot{y}^{2} .
\end{aligned}
$$

By a similar reason as above we can omit the case where $C_{1}=0, A_{2}=0$, $A_{1} \neq 0, C_{2}=0$.

Case VII. All coefficients are zero.

$$
\begin{aligned}
& \ddot{x}=0, \\
& \ddot{y}=0 .
\end{aligned}
$$

These are the canonical forms of the equations (5). The corresponding non-homogeneous forms are as follows:

$$
\begin{array}{rlrl}
y^{\prime \prime}+y^{\prime 3}-b y^{\prime 2}+a y^{\prime}-1 & =0, \\
y^{\prime \prime} . \pm \pm y^{\prime 2}+a y^{\prime}-1 & =0, \\
y^{\prime \prime} & \cdot y^{\prime}-1 & =0, \\
y^{\prime \prime} & -1 & =0, \\
y^{\prime \prime} & -y^{\prime 2}+y^{\prime} & =0, \\
y^{\prime \prime} & -y^{\prime 2} & & =0, \\
y^{\prime \prime} & & =0 .
\end{array}
$$

\section{§ 3. Canonical forms of the differential equations (continued).}

4. If we transform the coordinates by a linear transformation:

$$
\begin{aligned}
& x^{\prime}=p x+\dot{q} y, \quad p s-q r \neq 0, \\
& y^{\prime}=r x+s y,
\end{aligned}
$$


- the parameters of the affine connexion obey the following transformation law

$$
\Gamma_{\cdot \beta \gamma}^{\prime \alpha}=A_{i}^{\alpha} A_{\beta}^{\prime j} A_{\gamma}^{\prime k} \Gamma_{j k}^{i}
$$

where we have put

$$
\begin{aligned}
& A_{1}^{1}=p, \quad A_{2}^{1}=q, \\
& A_{1}^{2}=r, \quad A_{2}^{2}=s, \\
A_{1}^{\prime 1}= & \frac{s}{p s-q r}, \quad A_{2}^{\prime 1}=\frac{-q}{p s-q r}, \\
A_{1}^{\prime 2}= & \frac{-r}{p s-q r}, \quad A_{2}^{\prime 2}=\frac{p}{p s-q r} .
\end{aligned}
$$

If we apply this transformation to (5), we get :

$$
\begin{aligned}
& A_{1}^{\prime}=\frac{1}{(p s-q r)^{2}}\left[\left(p A_{1}+q A_{2}\right) s^{2}+\left(p C_{1}+q C_{2}\right) r^{2}\right] \\
& C_{1}^{\prime}=\frac{i}{(p s-q r)^{2}}\left[\left(p A_{1}+q A_{2}\right) q^{2}+\left(p C_{1}+q C_{2}\right) \dot{p}^{2}\right], \\
& B_{2}^{\prime}=\frac{-1}{(p s-q r)}\left[\left(r A_{1}+s A_{2}\right) q s+\left(r C_{1}+s C_{2}\right) p r\right] \\
& \text { etc., }
\end{aligned}
$$

where we have put

$$
A_{1}{ }^{\prime}=-\Gamma_{11}^{\prime 1}, \quad C_{1}^{\prime}=-\Gamma_{22}^{\prime 1}, \quad B_{2}{ }^{\prime}=-\Gamma_{12}^{\prime 2}, \quad \text { etc. }
$$

Now, consider the differential equations of the type II :

$$
\begin{aligned}
& \ddot{x^{2}}=a \dot{x}^{2}, \\
& \ddot{y}=\dot{x}^{2} \pm y^{2}
\end{aligned}
$$

Then, we'can easily see that

$$
A_{1}=\alpha, \quad C_{1}=0, \quad A_{1}=1, \quad C_{2}= \pm 1 .
$$

Let us study whether equations of the type $\left(9^{\prime}\right)$ with $a \neq=0$ are reducible . to the form : 


$$
\begin{aligned}
& \ddot{x^{\prime}}=0, \\
& \ddot{y}^{\prime}=A_{2}{ }^{*} \dot{x}^{\prime 2}+C_{2}{ }^{*} \dot{y}^{\prime 2}
\end{aligned}
$$

or not. If we put (10), into (8), we see that $C_{1}^{\prime}$, becomes zero when wé put $q=0$. Moreover, when $q=0$, we get.

$$
A_{1}^{\prime}-2 B_{2}^{\prime}=\frac{ \pm 2 r+a s}{p s}
$$

Hence, if we put

$$
q=0, \quad \frac{r}{s}=\mp \frac{a}{2},
$$

$C_{1}^{\prime}$ and $A_{1}^{\prime}=2 b_{2}^{\prime \prime}$ become both zero. Accordingly, by an appropriate projective change of affine connexion, it is possible to reduce our equations of paths to the desired form.

Now the curvature tensor of the affine connexion defined by (5) is easily calculated from the formula:

$$
R_{j k l}^{i} \stackrel{:}{=} \frac{\partial \Gamma_{j k}^{i}}{\partial x^{l}}-\frac{\partial \Gamma_{j l}^{i}}{\partial x^{k}}+\Gamma_{h l}^{i} \Gamma_{j k}^{h}-\Gamma_{h k}^{i} \Gamma_{j l}^{h}
$$

and givec us

$$
\begin{array}{ll}
R_{112}^{1}=C_{1} A_{2}, & R_{212}^{1}=-A_{1} C_{1}, \\
R_{112}^{2}=C_{2} A_{2}, & R_{212}^{2}=-A_{2} C_{1},
\end{array}
$$

the other components being all zero.

(12) show that the affine connexion defined by equations of the type II is not affinely flat. Hence ${A_{2}}^{*}$ and $C_{2}{ }^{*}$ can not vanish. Consequently, our equations are reducible to the canonical form:

$$
\begin{aligned}
& \ddot{x}=0, \\
& \ddot{y}=\dot{x}^{2} \pm \dot{y}^{2} .
\end{aligned}
$$

5. In the next place, let us consider the equations of the type I :

$$
\begin{aligned}
& \ddot{x}=a \dot{x}^{2}+\dot{y}^{2}, \\
& \ddot{y}=\dot{x}^{2}+\dot{b} \dot{y}^{2} .
\end{aligned}
$$


Putting

$$
A_{1}=\alpha, \quad C_{1}=1, \quad A_{1}=1, \quad C_{2}=b,
$$

into $(6)_{2}$, we see that $C_{1}^{\prime}$ becomes zero when and only when

$$
p^{3}+b p^{2} q+a p q^{2}+q^{3}=0 .
$$

Evidently there exist real values of $p$ and $q$ satisfying this equation. We denote these values by $p_{1}$ and $q_{1}$ and take arbitrary real, values $r_{1}$ and $s_{1}$ so as to satisfy

$$
p_{1} s_{1}-q_{1} r_{1} \neq 0
$$

Then by the transformation (7), $C_{1}^{\prime}$ becomes zero.

Accordingly, if we perform a suitable projective change of affine connexion, the equations of the type I reduce to the form

$$
\begin{aligned}
& \ddot{x^{\prime}}=A_{1}{ }^{*} \dot{x}^{\prime 2}, \\
& \ddot{y^{\prime}}=A_{2} \dot{x}^{\prime 2}+C_{2} * \dot{y}^{\prime 2},
\end{aligned}
$$

where $A_{2}{ }^{*}$ and $C_{2}{ }^{*}$ can not vanish in virtue of the same reason as above. Hence they are reducible to the canonical form II and consequently to the . canonical form $\mathrm{II}_{0}$. Thus we have proved the following lemma:

Lemma. The equations of the type $I$ and II are alivays reducible to the canonical form $I I_{0}$ by applying suitable coordinate transformations and projective changes of affine connexions.

Consequently all the equations of paths in considerations are reduced to one of the canonical forms of the type $\mathrm{II}_{0}$, III, IV, V, VI and VII.

\section{\$4. The possibility of binding any two points in plane by a path.}

6. Now the parameters of an affine connexion vary by coordinate transformations as follows:

$$
\Gamma_{j k}^{i} \frac{\partial x^{\prime \alpha}}{\partial x^{i}}=\frac{\partial^{2} x^{\prime \alpha}}{\partial x^{j} \partial x^{k}}+\Gamma_{\beta \gamma}^{\alpha} \frac{\partial x^{\prime \beta}}{\partial x^{j}} \frac{\partial x^{\prime \gamma}}{\partial x^{k}}
$$

If the given system of paths is affinely flat, coordinate system $x$, can be taken so that in the new coordinate system all paths can be represented 
analytically by linear equations of coordinates $x^{\prime \alpha}$. These coordinate systems are characterized analytically by those for $\cdot$ which $I_{{ }_{\beta} r}^{\mu_{\alpha}}=0$. Hence, in our two dimensional problem the tranformation functions $x^{\prime}=x^{\prime}(x, y), y^{\prime}=y^{\prime}$ $(x, y)$ are characterized as a pair of solutions of the following system of partial differential equations:

$$
\begin{aligned}
& \frac{\partial^{2} \phi}{\partial x^{2}}=-A_{1} \frac{\partial \phi}{\partial x}-A_{2} \frac{\partial \phi}{\partial y}, \\
& \frac{\partial^{2} \phi}{\partial x \partial y}=-B_{1} \frac{\partial \phi}{\partial x}-B_{2} \frac{\partial \phi}{\partial y}, \\
& \frac{\partial^{2} \phi}{\partial y^{2}}=-C_{1} \frac{\partial \phi}{\partial x}-C_{2} \frac{\partial \phi}{\partial y}
\end{aligned}
$$

7. Although we are considering of binding arbitrary points $\left(x_{0}, y_{0}\right)$ and $\left(x_{1}, y_{1}\right)$ each other by, a path, it will be sufficient to discuss only the possibility of binding the origin with an arbitiary points $\left(x_{1}, y_{1}\right)$ by a path. For the set of differential equations (1) is invariant under any translation

$$
x^{*}=x+\alpha, \quad y^{*}=y+\beta,
$$

- where $\alpha$ and $\beta$ are arbitrary constants.

Using these facts we shall study the possibility of binding, any two points in plane by a path of the given system.

8. Case VII. In this case, evidently, any two points in plane can be bound by a path (straight line).

Case VI. From (12) we see that the system of paths of the type VI is affincly flat. The set of equations (13) reduces in this case to

$$
\frac{\partial^{2} \phi}{\partial x^{2}}=0, \quad \frac{\partial^{2} \phi}{\partial x \partial y}=0, \quad \frac{\partial^{2} \phi}{\partial y^{2}}=-\frac{\partial \phi}{\partial y} .
$$

Hence we can take, as a pair of solutions,

$$
x^{\prime}=x, \quad y^{\prime}=\iota^{-y} .
$$

In the new coordinate system. $\left(x^{\prime}, y^{\prime}\right)$, the paths are straight.lines and the whole plane $(x, y)$ is mapped onto the upper half plane $y^{\prime}>0$. The image of the origin of the $(x, y)$ plane is the point $(0,1)$ in the $\left(x^{\prime}, y^{\prime}\right)$ plane. Hence, the problem whether the origin and any point in the $(x, y)$ plane can be bound by a path or not is equivalent to the problem: whether the 
point $(0,1)$ and any point $\left(x^{\prime}, y^{\prime}\right) y^{\prime}>0$ can be bound or not by a straight line in the upper half plane $y^{\prime}>0$, which is evidently possible. Consequently, in the Case VI the answer of our boundary value problem is affirmative.

Case V. The system of paths of the type $\mathrm{V}$ being also affinely flat, the set of equations (13) reduces in this case to

$$
\frac{\partial^{2} \phi}{\partial x^{2}}=-\frac{\partial \phi}{\partial x}, \quad \frac{\partial^{2} \phi}{\partial x \partial y}=0, \quad \frac{\partial^{2} \phi}{\partial y^{2}}=-\frac{\partial \phi}{\partial y}
$$

Hence we can take, as a pair of solutions,

$$
x^{\prime}=e^{-x}, \quad y=e^{-y} .
$$

In the new coordinate system $\left(x^{\prime}, y^{\prime}\right)$, paths are straight lines and the whole plane $(x, y)$ is mapped onto the first quadrant $x^{\prime}>0, y^{\prime}>0$. The image of origin of the $(x, y)$ plane is the point $O(1,1)$ in the $\left(x^{\prime}, y^{\prime}\right)$ plane. Hence by the same reasoning as in. Case VI, we can conclude that any two points in the $(x, y)$ plane can be bound by a path.

Case IV. The system of paths of the type IV is also affinely flat. The set of equations (13) reduces in this case to

$$
\frac{\partial^{2} \phi}{\partial x^{2}}=-\frac{\partial \phi}{\partial y}, \quad \frac{\partial^{2} \phi}{\partial x \partial y}=0, \quad \frac{\partial^{2} \phi}{\partial y^{2}}=0
$$

Hence, we can take, as a pair of solutions,

$$
x^{\prime}=x, \quad y^{\prime}=-\frac{x^{2}}{2}+y .
$$

The whole plane $(x, y)$ is mapped onto the whole plane $\left(x^{r}, y^{\prime}\right)$ one-toone and continuously. The origins on both planes correspond to each other. In the new coordinate system $\left(x^{\prime}, y^{\prime}\right)$, paths are straight lines. By the same reasoning as in cases" VI and V, we can conclude that any two points in $(x, y)$ plane can be bound always by a path.

Case III. The system of paths of the type III is also affinely flat. The set of equations (13) reduces in this case to

$$
\frac{\partial^{2} \phi}{\partial x^{2}}=-\frac{\partial \phi}{\partial x}-\frac{\partial \phi}{\partial y}, \quad \frac{\partial^{2} \phi}{\partial x \partial y}=0, \quad \frac{\partial^{2} \phi}{\partial y^{2}}=0 .
$$

We can take, as a pair of solutions, 


$$
x^{\prime}=e^{-x}, \quad y^{\prime}=-x+y .
$$

The whole plane $(x, y)$ is mapped onto the right half plane $x^{\prime}>0$ one-toone and continuously. The origin of the $(x, y)$ plane is transformed to the point $(1,0)$ on the $\left(x^{r}, y^{\prime}\right)$ plane. In the new coordinate system $\left(x^{\prime}, y^{\prime}\right)$, paths are straight lines. Hence by the same reasoning as in cases VI, $\mathrm{V}$, and IV, we can conclude that any two points in $(x, y)$ plane can . be bound always by a path.

9. Case $\mathrm{II}_{0}$

$$
\begin{aligned}
& \ddot{x}=0, \\
& \ddot{y}=\dot{x}^{2}+\varepsilon \dot{y}^{2}, \quad \varepsilon= \pm 1 .
\end{aligned}
$$

In this case the corresponding affine connexion is not flat. Indeed its curvature tensor becomes

$$
R_{112}^{2}=\varepsilon \text { and the others are all zero. }
$$

However, if we apply a projective change of affine connexion (4) with $\phi_{1}=0, \phi_{2}=\varepsilon$ the equations of paths $\mathrm{II}_{0}$ reduce to

$$
\begin{aligned}
& \ddot{x}=a \dot{x}^{2}-2 \varepsilon x y, . \\
& \ddot{y}=\dot{x}^{2}-\varepsilon \dot{y}^{2},
\end{aligned}
$$

which show that the affine connexion is flat, as we can easily verify it.

The set of equations (13) reduces in this case to

$$
\frac{\partial^{2} \phi}{\partial x^{2}}=-\frac{\partial \phi}{\partial y}, \quad \frac{\partial^{2} \not}{\partial x \partial y}=\varepsilon-\frac{\partial \phi}{\partial x}, \quad \frac{\partial^{2} \phi}{\partial y^{2}}=\varepsilon \frac{\partial \phi}{\partial y} .
$$

We can easily see that the general solution of the last set of equations is

$$
\phi=\alpha(x) c^{\varepsilon y}+\beta,
$$

where $\beta$ is a const. and $u(x)$ is a solution of the equation

$$
u^{\prime \prime}+\varepsilon u=0 \text {. }
$$

10. Case $\varepsilon=+1$. In this case a pair of solutions of (14) is given by

$$
x^{\prime}=e^{y} \sin x, \quad y^{\prime}=e^{y} \cos x .
$$

Every other solution is given as a linear combination of them : 


$$
\lambda x^{\prime}+\mu y^{\prime}+\nu,
$$

the coefficients being all constants.

Now, in the new coordinate system $\left(x^{\prime}, y^{\prime}\right)$, the paths are straight lines. However, in this case there happens somewhat different circumstance. from before.

Consider the strip between two straight lines both parallel to $y$-axis and passing through the points $(-\pi, 0)$ and $(\pi, 0)$ respectively. Then the strip is mapped one-to-one and continuously onto the whole $\left(x^{\prime}, y^{\prime}\right)$ plane with the exception of the negative $y^{\prime}$-axis. Indeed, the points on both boundary parallel lines of the strip are mapped onto the points of negative $y^{\prime}$-axis: Moreover, the origin of $\left(x^{\prime}, y^{\prime}\right)$ plane is the image of points at infinity $y=-\infty,(x$ : arbitrary $)$ of $(x, y)$ plane.

Accoridingly, the problem whether the origin and any point in (and not on the boundary of) the strip can be bound by a path or not is equivalent to the problem whether the point $(0,1)$ (image of the origin in $(x, y)$ plane) and a point (not on the negative $y^{\prime}$-axis) can be bound by a straight line or not, which does not pass through the origin. This is evidently possible. But, the origin and any point on the boundary of the strip in $(x, y)$ plane can not be bound by a path. For, it is equivalent to inquire whether the point $(0,1)$ and a point on the negative $y^{\prime}$-axis can be bound by a straight line or not, which does not pass through the origin. We can not allow the straight line segment between these two points pass through the origin of the $\left(x^{\prime}, y^{\prime}\right)$-plane, for the origin is the image of the points at infinity of $(x, y)$-plane. This is evidently impossible. Hence we can not bind the origin and any point on the boundary of the strip in $(x, y)$-plane by a path. Moreover, we can not bind the origin with any point outside of "the strip, too. It is clear that this fact does not alter when we take any other pair of solutions of (14),

Case $\varepsilon=-1$. In this case a pair of solutions of (14) is given by

$$
x^{\prime}=e^{x-y}, \quad y^{\prime}=-x-y .
$$

The whole plane is mapped onto the first quadrant $x^{\prime}>0, y^{\prime}>0$ one-to-one and continuously. In the new coordinate system $\left(x^{\prime}, y^{\prime}\right)$, paths are straight lines. Hence by the same reasoning as in cases III-IV we can conclude that any two points in the $(x, y)$-plane can always be bound by a path.

Summing up the results obtained in $n^{\circ} 8$ and $n^{\circ} 10$ we can recognize the truth of our theorem. 


\section{§5. A remark on the geometry of paths in the large.}

11. In the theory of geodesics in the large, we know that any two points on a closed surface of any genus can always be bound at least by one geodesic which is homotopic to any preassigned curve passing through the given two points. However, this fact does not hold in general for systems of paths even for the case of constant coefficients $A_{1}, B_{1}, \ldots \ldots, C_{2}$. To see this, let us take the system of paths of the type $\mathrm{II}_{0}$ with $\varepsilon=+1$. Devide the $(x, y)$ plane into a set of rectangles by two sets of equidistant parallel lines including $x$-axis and $y$-axis respectively. We can regard the $(x, y)$ plane as the universal covering surface of a torus endowed with a system of paths of the type $\mathrm{II}_{0}$ with $\varepsilon=+1$. Then, on account of the fact proved in $n^{\circ} 10$, we can easily see the truth of our assertion.

Mathematical Institute

Tôhoku University. 